Depolarisation, vermöge deren das Potential unter $\operatorname{den} C \ddot{u}$-Potentiale gehalten wird, erklärt werden.

Amerikanisches Patent Nr. 684205 vom 8. Oktober Igor und $\mathrm{Nr} .692507$ vom 20. Juni I90I beschreiben endlich die Kombinationen Zink - alkalisches Zinkat-, resp. Kadmium-AlkaliNickelsuperoxyd

Ein deutsches Patent, den Edison-Akkumulator betreffend, liegt nicht vor, der Thatsache entsprechend, dass das Wesentliche der Erfindung nicht neu ist.

Durch D. R.-P. Nr. I.28974 vom II. 8. oI Jässt sich v. Michalowski die Verwendung von dem nur schwach ätzende Wirkungen aufweisenden Kalium - oder Natriumaluminat statt der reinen Lauge schützen. In der Aluminatlösung geht Schwammzink anodisch nicht in Lossung. Der Elektrolyt bleibt also bei Bethätigung unverändert, da das sich anodisch primär abscheidende Hydroxyd stets durch das Alkali der Kathodenseite wieder gelöst wird. Der von vornherein grosse Widerstand des Elektrolyten dürfte sich bei Entnahme starker Ströme durch Hydroxydbildung ausserordentlich steigern, auch dürfte die Polarisation sehr hohe Werte annehmen.

\title{
GALVANOTECHNIK.
}

Ueber den Ersatz des Mononatriumsulfits durch Dinatriumsulfit in cyankalischen Kupferbädern. A. F is cher. Elektrochem. Zeitschr. 8, 192-193 (I90I). Man erbält nach Pfanhauser einen sehr schönen glänzenden Kupferüberzug auf Eisen und Zink mit folgenden Elektrolyten: I Liter $\mathrm{H}_{2} \mathrm{O}, 20 \mathrm{~g} \mathrm{NaHSO}_{3}, 20 \mathrm{~g}$ $\mathrm{Na}_{2} \mathrm{CO}_{3}$, $30 \mathrm{~g} \mathrm{Cu}(\mathrm{CN})_{2} \mathrm{KCN}$ und I g $\mathrm{KCN}$. Die beiden Salze $\mathrm{NaHSO}_{3}$ und $\mathrm{Na}_{2} \mathrm{CO}_{3}$ werden vorher gemischt und reagieren unter Kohlensäureund $\mathrm{Na}_{2} \mathrm{SO}_{3}$-Bildung. Verf. suchte diese vorbereitende Manipulation zu vermeiden, indem er $\mathrm{Na}_{2} \mathrm{SO}_{3}$ zusetzte, erhielt aber mit dem so hergestellten Elektrolyten schlechte Ueberzüge. Er schloss richtig, dass die Reaktion

$2 \mathrm{NHSO}_{3}+\mathrm{Na}_{2} \mathrm{CO}_{3}=2 \mathrm{Na}_{2} \mathrm{SO}_{3}+\mathrm{CO}_{2}+\mathrm{H}_{2} \mathrm{O}$ nicht vollständig verlaufe, setzte ausser dem Dinatriumsulfit noch Mononatriumsulfit hinzu, und erhielt sofort wieder den guten Niederschlag. Auch etwas Schwefelsäure thut dieselben Dienste. Er schreibt folgendes Bad vor, das einfacher herzustellen ist als das Pfanhausersche.

\section{Liter Wasser:}

\begin{tabular}{|c|c|}
\hline $\begin{array}{rl}20 \mathrm{~g} & \mathrm{Na}_{2} \mathrm{SO}_{3} \text { (calc.) } \\
6 & \mathrm{NaHSO}_{3} \\
20 & \mathrm{Na} \mathrm{SO}_{2} \mathrm{SO}_{4} \text { (calc.)) } \\
20 & \end{array}$ & $\begin{array}{l}\text { gelöst in einem } \\
\text { Teil des Wassers, }\end{array}$ \\
\hline & \\
\hline
\end{tabular}

Stromdichte 0,003 bis $0,004 \mathrm{Amp} / \mathrm{qcm}, 2$ bis 2,5 Volt, etwa I5 $\mathrm{cm}$ Elektrodenentfernung.

H. D.

\section{EINRICHTUNG UND ZIELE EINER BUNSEN-GESELLSCHAFT. \\ Von E. Jordis.}

Die Frage, ob sich unsere Gesellschaft $z u$ einer Bunsen-Gesellschaft erweitern solle, ist durch die bisher erfolgten Erörterungen bejaht worden; weniger geklärt scheint nir die Frage: Wie soll diese Erweiterung erfolgen? Mit welchen augenblicklichen und zukünftigen tielen?

Als Hauptgesichtspunkte wurden bisher betont: T. Die Unmöglichkeit, im engen Rahmen der speziellen Elektrochemie zu hleiben, zumal derselbe ja schon fortgesetzt durchbrochen werde. 2. Die Herbeiführung einer besseren gegenseitigen Verständigung von Theorie und Praxis. Melur nebenbei gestreift wurde 3 . die Fülle der zeitgenössischen Litteratur und 4. die Verhinderung weiterer vorzeitiger Vermehrung derselben durch neve Spezialzeitschriften.

ad I. In der Diskussion ist mit vollem Recht betont worden, dass es eigentlich nicht nötig sei, den Namen der Gesellschaft zu ändern, da schon früher auch nicht speziell elektrochemische Bestrebungen bei uns reges Interesse fanden. Ich bin auch der Meinung, dass im Begriffe "Elektrochemie" die unerlässlichen Vor- bedingungen, nämlich die Pflege sowohl der , reinen Chemie" als auch der Physik, besonders der Elektrizitätslehre und der physikalischen Chemie, schon ohne weiteres eingeschlossen sind. Trotzdem aber halte ich es für wünschenswert, einen grösseren Verband zu schaffen, innerhalb dessen die, deutsche elektrochemische Gesellschaft" unverändert weiter wirken könnte.

ad 2. In diesem Verbande wird sich dann anch durch geeignete Mittel die engere Fühlung mit der Technik erreichen lassen. Hierüber bestehen aber anscheinend manchmal nicht ganz zutreffende Vorstellungen! Die in beiderseitigem Interesse sehr wünschenswerte Annäherung kann immer nur so zu stande kommen, dass der wissenschaftliche Forscher seine Ergebnisse in einer allgemein verständlichen Form zur Kenutnis bringt und selbst betont, welcher Zweig der Technik seiner Meinung nach aus diesen Forschungen Nutzen ziehen könnte.

ad 3. Die Bemerkung des Herrn Ostwald, dass eigentlich schon die Zeitschrift für physikalische Chemie allein einen Umfang besitzt, der es schwer macht, die 
Veröffentlichungen dauernd und gründlich zu verfolgen, hatte für viele einen komischen Beigeschmack. Indessen ist sie leider nur zu berechtigt! Die Ueberfülle der Litteratur ist zur Zeit eine erdrückende, so dass sie geradezu zum Henmnis einer Entwicklung der Wissenschaft nach der allgemeinen Seite wird. Und doch ist die Ableitung allgemeiner Gesichtspunkte die Vorbedingung zur Ausbildung jener Theorieen, welche die anorganische Chemie zu ähnlicher Blüte bringen sollen, in welcher die organische jetzt steht.

Fasst man nur die Zeitschriften ins Auge, welche man in wissenschaftlichen Laboratorien $z u$ finden gewohnt ist, nämlich z. B. die Berliner Berichte, Zeitschrift für angewandte Chemie, für Elektrochemie, für anorganische Chemie, für analytische Chemie, Liebigs Annalen, Chemiker Zeitung u. s. w. und quasi als Generalregister zu allen das Chemische Centralblatt, ferner die verschiedenen Jahrbücher, Sammlungen und Encyklopädieen, so hat man damit ein dem Forscher regelmässig gegenübertretendes Material, dessen Bewältigung vollkommen unmöglich ist. Dabei sind ausländische Zeitschriften noch gar nicht berücksichtigt, obgleich gerade für unsere speziellen Bestrebungen auch diese wohl beachtet werden müssen. Es ist schon schwierig, sich aus den Registern dieser periodischen Erscheinungen das für das eigene Forschungsgebiet wichtige herauszusuchen und eingehender durchzunehmen.

Nun sehen wir aber noch fortwährend neue Zeitschriften, darunter auch ausgezeichnete Blätter, entstehen! Die notwendige Folge ist: immer weitergehende Zersplitterung, das Ueberwuchern von Spezialtheorieen für allerlei kleinere Gebiete und die Vernachlässigung allgerneiner grosser Gesichtspunkte, wodurch wieder die gegenseitige Befruchtung naheliegender Gebiete mehr und mehr aufhört. So geht fortwährend eine Unmenge geistiger Energie verloren, die bei systematischer $\mathrm{Zu}$ sammenarbeit wertvollste Resultate zeitigen könnte.

ad 4. Daher ist auch der Gedanke, die Schaffung neter Zeitschriften so lange als möglich zu verhindern, freudigst $z u$ begrüssen. Ja man wird ihn dahin erweitern dürfen, dass die Verschmelzung bestehender anzustreben wäre.

Ist man sich über die entwickelten Wünsche, Schwerigkeiten und Missstände klar, so liegt auch der Weg für die Erfüllung, bezw. Abhilfe vorgezeichnet.

Da man die Spezialisierung nicht hindern kann, so muss man sie systematisch organisieren, indem man aber zugleich dafür sorgt, dass durch den Forscher selbst oder durch besondere dazu geschaffene Organe die allgemein wichtigen Resultate auch Fernerstehenden in allgemein verständlicher Form bekannt gegeben werden und so anregend und befruchtend wirken können. Damit wäre schon eine Hauptschwierigkeit beseitigt, die zwischen den Vertretern verschiedener Fächer, z. B. der Technik und der reinen Theorie, vielfach vorliegen, dass nämlich beide einander nicht verstehen und den Punkt nicht finden können, in dem ihre Interessen zusammentreffeu.

Wie ist das nun zu erreichen?
Schon jetzt besteht eine gewisse geordnete Spezialisierung, der nicht nur unsere Gesellschaft, sondern auch eine Anzahl anderer Vereine und ausserdem eine Reihe bedeutender, schon genannter Spezialzeitschriften ihr Bestehen verdanken. Würde es möglich sein, diese Faktoren für die mir vorschwebende, umfangreiche, wissenschaftliche, auf ein $\mathrm{Ziel}$ gerichtete Arbeit zu interessieren, und all die Kräfte, die jetzt teils gleichgültig nebeneinander, teils mehr oder weniger in Kampf gegeneinander zersplittert sind, zus ammenzufassen, so würde sich eine sehr zeitsparende Vereinigung aller zusammengehörigen Arbeiten, kritische Berichterstattung über solche, welche, anderen Fächern entstammend, für die verschiedenen Spezialgebiete nach der oder jener Richtung Wert haben und fortlaufende Orientierung über wesentlich grössere Gebiete der Gesamtchemie, als sie jetzt dem Einzelnen möglich ist, unschwer erreichen lassen. Wie das zu erreichen wäre, soll hier nicht weiter erörtert werden; die Mittel liegen ausserdem zum Teil sehr nahe und offensichtlich da!

Mein Vorschlag geht also dahin, die ,BunsenGesellschaft" zu gründen mit der ganz bestimmten Absicht, in ihr alle Bestrebungen auf dem Gebiete der Chemie im weitesten Sinne, mögen sie schon bestehen oder sich erst später entwickeln, zu vereinigen, soweit sie sich zusammenschliessen wollen. Daher würde ich auch keine "Deutsche Bunsen-Gesellschaft" wünschen! Denn warum sollen uns z. B. amerikanische oder russische Vereinigungen unwilkommen sein, wenn sie gleichen Bestrebungen huldiget?

Im Rahmen der „Bunsen-Gesellschaft“ hätten wir dann als vorläufig erste die „, Deutsche Elektrochemische Gesellschaft". Neben dieser wäre Platz für alle anderen! Die Gesamtheit aller Publikationen würde die ,Abhandlungen der Bunseu-Gesellschaft" bilder. Ein besonderes Organ oder mehrere würde, wie gesagt, bestimnt sein, aus allen Gebieten zusammenfassende Darstellungen zu bringen, die allen Chemikern von Interesse sind. Das geschieht ja auch schon jetzt, aber mit völliger Zersplitterung der Kräfte und Mittel. Auch hier brauchte es nur des Zusammenschlusses, um viel Arbeit, viel Druckerschwärze, grosse Kosten $z$ u ersparen und $m$ it den so gewonnenen Mitteln Grosses zu erreichen.

Der neue Titel unserer Zeitschrift ergiebt sich danach von selbst als: „Abhandlungen der BunsenGesellschaft" und darunter: "Z eitschrift für Elektrochemie und angewandte physikalische Chemie“. Den Titel ,Berichte der Bunsen-Gesellschaft" halte ich nicht für gut gewählt, da die Abkürrung Bunsen-Berichte (B. B.) zu leicht Verwechslungen mit den Berliner Berichten hervorrufen kann, was bei Bunsen-Abhandlungen (B. A.) vermieden wird. Ausserdem schreibt der Forscher heutzutage nicht mehr Berichte an seine Gesellschaft, sondern Abhandlungen.

Was von meinen Anregungen alsbald verwirklicht werden kann, deckt sich vielfach mit den Ausführungen von anderer Seite; was noch wie Zukunftsmusik klingt, dürfte doch Beachtung verdienen; manches, z. B. die Zusammenarbeit mehrerer Gesellschaften und Zeit- 
schriften, könnte schon jetzt angebahnt werden. Was? und wie das Einzelne? zu geschehen hätte, steht aber heute nicht zur Diskussion.

Nachschrift. Auch den sehr beachtenswerten Ausfïhrungen des Herrn Buchner in Nr. I6 würde mein Vorschlag gerecht werden. Aus denselben geht m. E. klar hervor, dass eine Vereinigung ganz verschiedener Dinge in einem Namen eben unausführbar ist, während die von mir vorgeschlagene Unterteilung das beibehält, was sich bewährt hat, und doch die erweiterten Ziele der Gesellschaft wie der Zeitschrift, jede an ihrem Orte, dentlich ausdrückt.

\section{HOCHSCHUL- UND PERSONAL-NACHRICHTEN.}

Mannheim. Der Seniorchef der Firma: ,Mannheimer Maschinenfabrik Molu $\mathrm{r}$ \& Federhaff", Kommerzienrat Hermann Mohr, ist am I5. April gestorben.

Tübingen. Prof. Dr. Freiherr von Pechmann,
Direktor des Chemischen Universitätslaboratoriums, ist in Alter von 5o Jahren gestorben.

Berichtigung zum Vorlesungsverzeichnis Seite 2Io. Leipzig. R. Luther liest über Stöchiometrie.

\section{NEUE B ÜCHER.}

The Elements of Physical Chemistry von H. C. Jones. XI und $56_{5}$ Seiten. Macmillan Co., New York 1902. Preis $4 \mathrm{Mk}$.

Trotzdem unsere deutsche Litteratur durch die bekannten klassischen Darstellungen von Ostwald, Nernst und ran't $H$ off in hervorragender Weise mit Darstellungen der physikalischen Chemie bedacht ist, so dass für uns das vorliegende Werk kaum in Betracht kommt, so ist fïr die englische Leserwelt das Buch zweifellos eine willkommene Gabe, denn es behandelt das ganze Gebiet in korrekter Weise und besitzt zwei grosse Tugenden, nämlich eine sehr leicht verständliche und eine sehr lebendige Darstellung. Auf den Inhalt des näheren einzugehen, erübrigt sich, nur sei bezüglich der Auswahl des Gebotenen die Meinung nicht unterdrückt, dass sie mitunter den objektiven Maassstab, wenn auch aus verzeihlichen Gründeı, vermissen lässt, Der Buchstabe J des Registers ist dafür bezeichtiend: Doch dies schmälert die Brauchbarkeit des nützlichen Buches nicht, dem in seiner Feimat eine recht grosse Verbreitung gewünscht sei.

\section{VEREINSNACHRICHTEN.}

\section{Deutsche Elektrochemische Gesellschaft. Hauptversammlung in Würzburg 1902.}

Unter Hinweis auf die in Heft 15 und 16 gegebenen Nachrichten wird für die Herren Teilnehmer noch mitgeteilt, dass die Geschäftsstelle der Versammlung sich am 8. Mai, abends voti 7 Uhr ab, im Russischen Hofe, an 9. und ro. Mai aber in Vorstandszimmer des Chenischen Universitäts - Laboratoriums, Pleicherring II, befindet.

Anmeldungen zur Mitgliedschaft sind satzungsgemäss an den ersten Vorsitzenden, Herrn Profess or Dr. valt't Hoff, Charlottenburg, Uhlandstr, 2, zu richten; die Anmeldungen müssen von einem Mitglied der Gesellschaft befürwortet sein.

Zahlungen werden ausschliesslich an den Schatzmeister, Herrn Dr. Marquart, BettenhausenCasse1, erbeten.

Alle anderen geschäftlichen Mitteilungen wolle man an die Geschäftsstelle der Deutschen Elektrochemischen Gesellschaft, Leipzig, Mozartstr. 7 . richten.

Die Versendung der Vereinszeitschrift geschieht durch die Verlagsbuchliandlung unter deren Verantwortlichkeit, und zwar nach dem Auslande unter Kreuzband, nach dem Inlande ohne solches, wie bei Tageszeitungen. Die Nachlieferung fehlender Nummern ist deshalb in Inlande möglichst bald bei der BestellPostanstalt nachzusuchen, ebenso ist diese - wie auch die Geschäftsstelle - von Adressenänderungen $z u$ benachrichtigen. Etwaige weitere Mitteilungen über fehlende Hefte, insbesondere vom Auslande, sind an die Geschäftsstelle zu richten.

An die neq eintretenden Herren Mitglieder wird die Vereinszeitschrift erst nach Zahlung des Mitgliedbeitrages geliefert.

\section{Aufgenommene Mitglieder.}

Nr. 863. Erlenbach, Dr. Arnold, Dessau.

" 864. Magnanini, Gaetano, Prof. Dr., Modena.

Anmeldungen für die Mitgliedschaft.

Gemäss $\$ 3$ der Satzungen werden hiermit die Namen der Herren, Firmen u. s. w., welche sich beim Vorstande für die Aufnahme gemeldet haben, veröffentlicht. Einspruch gegen die Aufnahme eines gemeldeten Mitgliedes ist innerhalb zweier Wochen (also bis zum 15. Mai einschliesslich) zu erheben.

Nr. 804. Rosenlew, E., Mag. Phil. aus Helsingfors, Zürich, Elektrochemisches Laboratorium des Polytechnikums; durch R. Lorenz.

" 805. Egli, Dr. J., Carbidfabrik Gurtnellen (Gotthardbahn); durch R. Lorenz.

" 806. Hahn, Oscar, Leipzig, Windmühlenstr. 23; durch Max Bodenstein.

" 807. Brand, Dr. Kurt, Giessen, Phys. - chem. Inst. der Universität; durch K. Elbs.

" 808. Briner, Emil, Docteur és sciences, CarougeGenf, Rue Joseph Girard; durch Ph. A. Guye. 